\title{
ELECTROSTATIC CHARGE ON SPRAY DROPLETS OF AQUEOUS SURFACTANT SOLUTIONS
}

\author{
M. Polat, ${ }^{* \dagger}$ H. Polat ${ }^{\ddagger}$ and S. Chander ${ }^{\S}$ \\ * Department of Mining Engineering, Dokuz Eylul University, Bornova-İzmir, Turkey \\ ‡ Department of Chemistry, İzmir Institute of Technology, Basmane-Ìzmir, Turkey \\ ${ }^{\S}$ Department of Energy and Geo-Environmental Engineering, Penn State University, University Park, USA
}

(First received 19 March 1999; and in final form 14 July 1999)

\begin{abstract}
Electrostatic charges on individual spray droplets were measured using a refined form of the Millikan oil drop method. The measurement system consisted of three main sections; a droplet generation cell, a settling column and a charge measurement chamber. The trajectories required for calculation of charge were determined using a high-speed motion analyzer coupled to a long-focallength microscope. Charges on droplets were manipulated by the addition of surface-active agents into the spray solution. Droplet charge was a function of the type and concentration of the surfactant added. For ionic surfactants, it showed a maximum at low surfactant concentrations, decreased with further surfactant addition and was constant after the CMC. The charge on cationic surfactants was always more than that observed with the anionic surfactants. Nonionic surfactants displayed a steady increase in droplet charge with increasing concentration. The charges were lower compared to the ionic surfactants. (C) 2000 Elsevier Science Ltd. All rights reserved.
\end{abstract}

\section{NOMENCLATURE}

$C_{\mathrm{d}}$ drag coefficient

$d^{*}$ dimensionless droplet diameter

$d$ droplet diameter

$E$ electric field strength

$F_{\text {net }}$ net force acting on droplet in horizontal direction

$F_{\mathrm{e}} \quad$ electric force in horizontal direction

$F_{\mathrm{d} x}$ drag force in horizontal direction

$g$ gravitational acceleration

$n$ number of droplets in population

$q$ net charge on droplet

$q_{i}$ charge on $i$ th droplet in population

$V_{y}^{*} \quad$ droplet's dimensionless terminal settling velocity

$V_{y}$ droplet's terminal settling velocity

$V_{x}$ droplet's horizontal velocity

$\rho_{\mathrm{a}}$ density of air

$\rho$ density of droplet

$\mu_{\mathrm{a}} \quad$ viscosity of air

\section{INTRODUCTION}

Droplets of pure water and electrolyte solutions that occur in nature (for example, rain and sea water) are spontaneously charged. Spontaneous charging of droplets can be caused by the development of a double layer due to the presence of ions dissolved in water (Lenard, 1892; Chapman, 1937, 1938a, b; Iribane and Mason, 1967; Bailey, 1988), statistical fluctuation of electrolytic ion concentration among the droplets (Chapman, 1934; Dodd, 1952), contact electrification between the solution and the surrounding material (Vick, 1953; Byrne, 1977) and capture of ions from air (White, 1951; Liu et al., 1987; Pearse and Pope, 1977; Polat et al., 1993, 1996). These mechanisms may act simultaneously in charging a droplet. Hence, the sign and the magnitude of the droplet charge is difficult to control and predict.

\footnotetext{
${ }^{\dagger}$ Author to whom correspondence should be addressed.
} 
Charged droplets can be created by electrospraying where a potential difference is applied to a cone or a capillary. These droplets have been observed to have very narrow size distributions. Since the total current flow through the system is measured, an average droplet charge can be calculated dividing the total current flow by the volume of spray solution (Yamashita and Fen, 1984; Gomez, 1993; Tang and Gomez, 1994a, b).

Presence of various species in the spray solution which adsorb at the droplet/air interface should be expected to affect the droplet charge. Spray painting, ink-jet printing, photocopying, crop spraying, dust suppression etc. are examples of industrial applications where such solutions are utilized. Studying the charging behavior of these types of spraying applications requires a direct measurement of charge on individual droplets since (i) the total charge in the system cannot be determined, (ii) the size distribution of the droplets can be quite wide.

The aim of this paper is to describe a method accurately measuring the electrostatic charges on individual spray droplets and to determine the effect of the presence of surface active substances in the spray solution on droplet charge.

\section{PRINCIPLES OF CHARGE MEASUREMENT}

Hopper and Laby (1941) and Kunkel (1950a, b, c) refined Millikan's measurements of the electronic charge Millikan (1913) by employing a horizontal electrical field. Our setup which is based on Hopper and Laby's includes three enhancements:

- A high-speed camera coupled to a telescopic microscope was used for tape-recording the droplets to determine their trajectories. This allowed the recording of a very large number of droplets (over 100 thousand), hence, better statistical accuracy.

- Terminal settling equations which were applicable for much wider size range were utilized to calculate the settling velocities.

- Sizes of the droplets could be directly measured since natural lighting was utilized. The observed sizes could then be employed to cross check the sizes calculated from the terminal settling equations. This was done earlier in the work and was not pursued later since the two sizes were observed to agree well.

The device works on the principle that a free falling, charged body will respond to an electrical field. The two opposing forces acting on such a body in a horizontal electrical field are the electric and drag forces. The net force will be zero for a droplet moving freely in an electric field, hence $F_{\mathrm{e}}=F_{\mathrm{dx}}$ where $F_{\mathrm{e}}=q . E$ and $F_{\mathrm{dx}}=\pi C_{\mathrm{d}} \rho_{\mathrm{a}} V_{\mathrm{x}}^{2} d^{2} / 8$. Then,

$$
q=\frac{\pi C_{\mathrm{d}} \rho_{\mathrm{a}} V_{\mathrm{x}}^{2} d^{2}}{8 E} .
$$

The dimensionless drag coefficient, $C_{\mathrm{d}}$ is a function of Reynolds number. It is given by Concha and his co-workers (Concha and Almendra, 1979a; Concha and Almendra, 1979b; Concha and Barrientos, 1982) for Reynolds numbers covering Stokes, intermediate and Newton regime as $C_{\mathrm{d}}=0.28\left(1+9.06 / \mathrm{Re}^{0.5}\right)^{2}$.

Solving equation (1) requires a knowledge of the diameter and the horizontal velocity of the droplet in an electrical field of known strength. The droplet diameter can be calculated from the vertical velocity using the equation given by Concha and his co-workers:

$$
d^{*}=0.07 V_{y}^{* 2}\left[1+\left(1+68.49 / V_{y}^{* 1.5}\right)^{0.5}\right]^{2},
$$

where $d^{*}$ is the dimensionless diameter and $V_{y}^{*}$ is the dimensionless settling velocity. The dimensionless parameters are related to the actual particle diameter and settling velocity as $d^{*}=d / P$ and $V_{y}^{*}=V_{y} / Q$ where $P=\left(3 \mu_{\mathrm{a}}^{2} / 4 \Delta \rho \rho_{\mathrm{a}} g\right)^{1 / 3}$ and $Q=\left(4 \Delta \rho \mu_{\mathrm{a}} g / 3 \rho_{\mathrm{a}}^{2}\right)^{1 / 3}$. It follows that if the horizontal and vertical velocity components of a droplet falling free in an electrical field is determined, the net charge carried by this droplet can be calculated. 


\section{EXPERIMENTAL SETUP}

The experimental setup consisted of three main sections (Fig. 1); a droplet generation cell where individual droplets were formed from surfactant solutions by spraying, a settling column where these droplets are allowed to reach terminal velocity, and a charge measurement chamber.

The droplet generation cell consisted of a rectangular box which enclosed a stainless-steel spray nozzle of model 1/8 JJ SS from Illinois Spraying Company. The nozzle had two inlet openings for the air and the solution. The air flow rate to the nozzle was kept constant at $4.21 \mathrm{~min}^{-1}$. The flow rate of the spray solution was around $2 \mathrm{ml} \mathrm{min}{ }^{-1}$.

In the experiments where the droplets were charged by applying a potential difference, a stainless-steel tube of $1.5 \mathrm{~cm}$ in diameter and $5 \mathrm{~cm}$ in length was attached in front
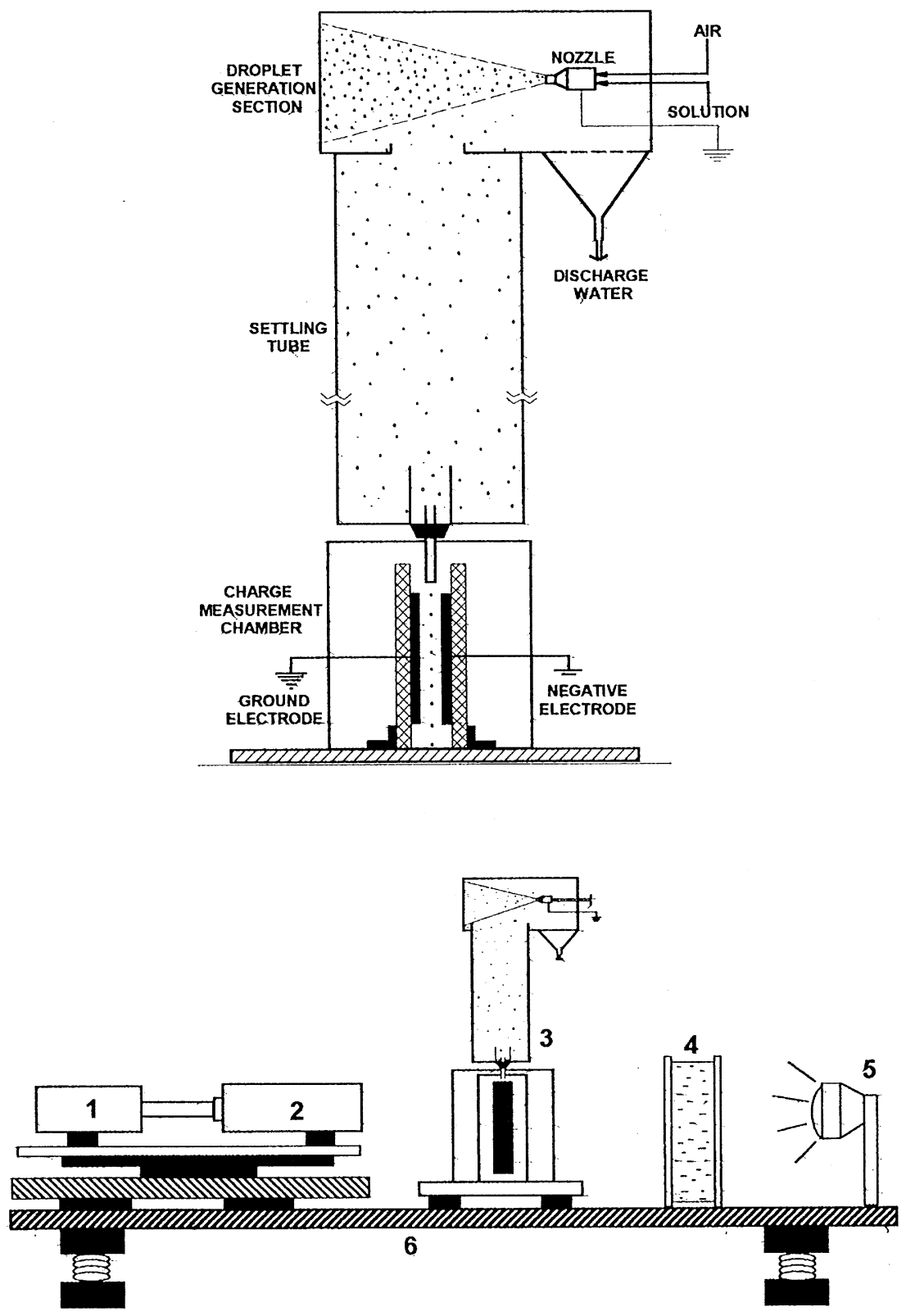

Fig. 1. A schematic view of the droplet charge analyzer and the set up used in measuring the droplet charge: (1) high-speed camera, (2) long-focal-length microscope, (3) the droplet charge analyzer, (4) water tank, (5) light source, (6) vibration free table. 
of the nozzle. The tube whose potential could be adjusted by a DC power supply was insulated from the nozzle using a plexiglas separator. In all the experiments the nozzle itself was grounded to prevent charging of the droplets by the contact potential effects.

After an initial impact with the walls of the droplet generation cell, some spray droplets fell into the plexiglas settling column. The settling times in the column varied between 2 to $3 \mathrm{~s}$. Two concentric, overlapping glass tubes, with inside diameters of 20 and $5 \mathrm{~mm}$, connected the settling column to the charge measurement chamber. The diameter of the smaller glass tube was large enough (about 70 times that of an average droplet) to prevent sampling errors.

The chamber contained two parallel stainless-steel rectangular electrodes, which were positioned vertically below the settling column. The plates were separated by a distance of $8.0 \mathrm{~mm}$. An electrical field of -3750 volt $\mathrm{cm}^{-1}$ was created between the electrodes using a DC high-voltage power supply. The system was placed in a Plexiglas box inside of which was lined with a grounded fine copper mesh to prevent stray electricity.

In order to record the trajectories of the micron-sized droplets required for the calculation of the electrostatic charge, the set up was placed in front of a long focal length microscope connected to a high speed motion analyzer (Fig. 1). A transparent water tank was placed between the light source and the analyzer to eliminate any temperature gradients. The whole setup was placed on a vibration free table.

The optical system was a Questar model telescopic microscope and the motion analysis system was a Spin Physics Model 2000 high-speed video camera recorder capable of taking pictures at a rate of 2000 frames s $^{-1}$.

\section{METHOD}

Before recording, the settling column was saturated with water vapor by spraying distilled water for at least $10 \mathrm{~min}$ to minimize static charges in the system and evaporation of droplets. The initial position of a droplet on a given video frame was determined by a built-in screen micrometer system in terms of $X$ and $Y$ coordinates. The picture was then advanced 10 frames, which corresponded to an elapsed time of $5 \mathrm{~ms}$, and the new coordinates of the droplet were determined. Dividing the distance between the two $X$ and $Y$ readings with the elapsed time gave the velocity of the droplet in both coordinates.

All of the experiments with a given surfactant were carried out within the same day starting from the lowest concentration. All the parts of the system were cleaned thoroughly between each run. A fresh surfactant solution was prepared before each experiment. The solution was constantly stirred and its temperature was kept at $22^{\circ} \mathrm{C}$ using a temperature bath.

\section{MATERIALS}

Some common characteristics of the surfactants used in preparing the solutions for the droplet charge experiments are given in Table 1. Structures of these surfactants are presented in Fig. 2.

Double distilled water with a resistivity of at least 1.5 Mohm was used in all the experiments to prepare the electrolyte and surfactant solutions. The distillation equipment was a Barnstead 210 biopure distilled water system which was equipped with a $\mathrm{Q}$ baffle system to produce pyrogen-free water.

\section{EXPERIMENTAL RESULTS}

An example output of the raw data produced from the charge measurements is given in Fig. 3 for distilled water. The figure shows that most of the droplets are positively charged. In order to obtain concise information from such data the results will be presented on the 
Table 1. Selected properties of the surfactants used in this study

\begin{tabular}{|c|c|c|c|c|c|}
\hline Commercial name & $\begin{array}{l}\text { Hydrophobic } \\
\text { group }\end{array}$ & $\begin{array}{l}\text { Hydrophilic } \\
\text { group }\end{array}$ & MW & $\begin{array}{l}\text { C.M.C. } \\
\left(\times 10^{-3} \mathrm{Mol} / \mathrm{l}\right)\end{array}$ & $\begin{array}{l}\text { Surface tension } \\
(\mathrm{mN} / \mathrm{m})\end{array}$ \\
\hline \multicolumn{6}{|l|}{ Anionic surfactants ${ }^{\mathrm{a}}$} \\
\hline $\begin{array}{l}\text { Na-lauryl Sulfate } \\
\left(\mathrm{NaLSO}_{4}\right)\end{array}$ & Lauryl & Sulfate & 288 & $8.25^{\mathrm{c}}$ & \\
\hline Aerosol-OT (AOT) & Dioctyl & Sulfosuccinate & 444 & $1.3^{\mathrm{d}}$ & $26.0^{\mathrm{d}}$ \\
\hline \multicolumn{6}{|l|}{ Cationic surfactants ${ }^{\mathrm{a}}$} \\
\hline Dodecyl amine (DAH) & Dodecyl & Amine & 222 & $13.3^{\mathrm{c}}$ & \\
\hline Coco amine (C5) & Coco & $\mathrm{PEO}^{\mathrm{b}}(5 \mathrm{Mol})$ & 425 & $0.16^{\mathrm{e}}$ & $33.0^{\mathrm{g}}$ \\
\hline Cocoamine $(\mathrm{C} 10)$ & Coco & PEO (10 Mol) & 645 & $0.16^{\mathrm{e}}$ & $38.0^{\mathrm{g}}$ \\
\hline Coco amine (C15) & Coco & PEO (15 Mol) & 825 & $0.16^{\mathrm{e}}$ & \\
\hline \multicolumn{6}{|l|}{ Nonionic surfactants ${ }^{\mathrm{a}}$} \\
\hline Triton X-100 (TX100) & Octyl phenol & PEO (9.5 Mol) & 628 & $0.22^{\mathrm{f}}$ & $30.1^{\mathrm{f}}$ \\
\hline Triton N-101 (TN101) & Nonyl phenol & PEO (9.5 Mol) & 642 & $0.033^{\mathrm{f}}$ & $30.0^{f}$ \\
\hline
\end{tabular}

${ }^{\text {a }}$ Commercial sources of these widely used surfactants are various. A detailed listing of the producers can be found in Ash and Ash (1992).

${ }^{\mathrm{b}}$ PEO-Poly ethylene oxide.

c (Mukerjee and Mysels, 1971).

d (Osipow, 1962).

${ }^{\mathrm{e}}(\mathrm{Hu}, 1992)$.

f (Mohal, 1988).

$\mathrm{g}($ Reck, 1967).

basis of few statistical parameters. These are:

The root-mean-square $(R M S)$ charge: This parameter shows the extent of charging for a population of droplets irrespective of sign. It is calculated according to the equation $\mathrm{RMS}=\left(\sum_{i=1}^{n} q_{i}^{2}\right)^{0.5} / n$.

Total positive or total negative charges: This parameter is obtained by separately summing the positive and negative charges in the system based on 200 droplets.

In the case of the distilled water, the RMS charge was $5.4 \times 10^{3}$ electron (e) and the total positive and negative charges were $629.8 \times 10^{3}$ and $-81.6 \times 10^{3}(\mathrm{e})$, respectively. For this population, the percentage of positively and negatively charged droplets were 67 and $12 \%$, respectively. The remaining $21 \%$ were neutral droplets.*

\section{Charge on droplets of surfactant solutions}

Anionic surfactants: Addition of the anionic surfactants increased the droplet charge at low concentrations significantly. The RMS charges for the anionic sodium lauryl sulfate $\left(\mathrm{NaLSO}_{4}\right)$ displayed a maximum at very low concentrations and decreased afterwards with further surfactant addition (Fig. 4). The RMS charge became almost as low as that of the distilled water at $3.5 \times 10^{-3} \mathrm{M}$ and stayed constant at concentrations above this. It should be noted that this concentration is very close to the CMC of this surfactant. The total negative charges displayed a behavior very similar to the RMS charge whereas the total positive charges seemed to be fluctuating at low values. This demonstrates that negative charges are dominant on the droplets generated from the solutions of this surfactant. This can be better seen from the percentages of the positively and negatively charged droplets. While the fraction of negatively charged droplets was significantly low in the case of distilled water $(12 \%)$, it was raised to a value of $77 \%$ upon addition of this negatively charged surfactant. The percentages became somewhat closer around the $\mathrm{CMC}$.

The other anionic surfactant, Aerosol-OT (AOT), showed similar charging character even though its droplets were less strongly charged than those of $\mathrm{NaLSO}_{4}$ at low concentrations

\footnotetext{
* The term "neutral" means those droplets with charges less than the resolution of the analyzer, about 100 (e).
} 


\section{SODIUM LAURYL SULFATE}

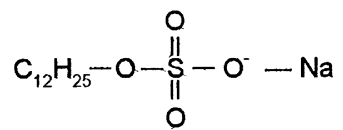

\section{AEROSOL-OT (SODIUM DIOCTYL SULFOSUCCINATE)}

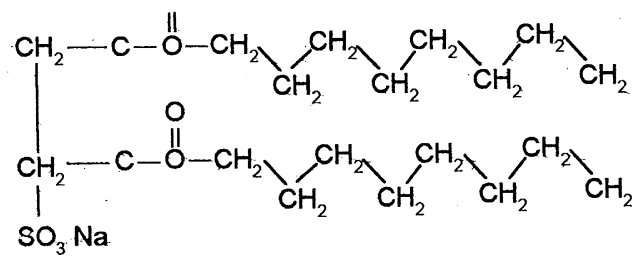

\section{DODECYL AMINE HYDROCHLORIDE}
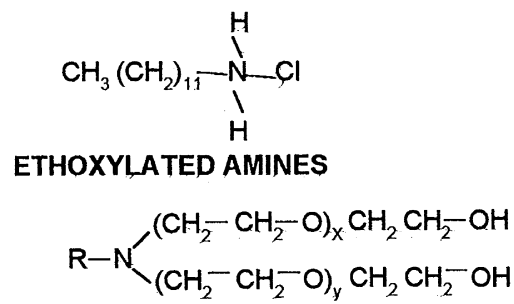

$x+y=$ Number of moles of ethylene oxide $(5,10$ ve15 for $C-5, C-10$ and $C-15$ $R=$ Number of atoms in hydrocarbon chain (12 for coco amines)

\section{ETHOXYLATED NONYL PHENOLS}

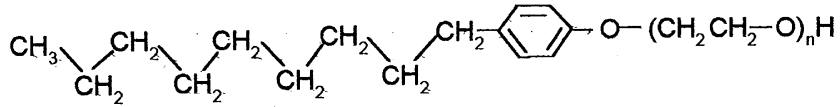

$\mathrm{n}=$ Number of moles of ethylene oxide $(9.5$ for $\mathrm{N}-100)$

\section{ETHOXYLATED OCTYL PHENOLS}

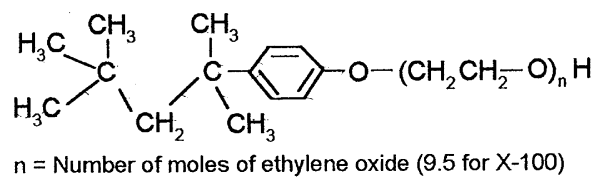

Fig. 2. Chemical structures of the surfactants employed in the study.

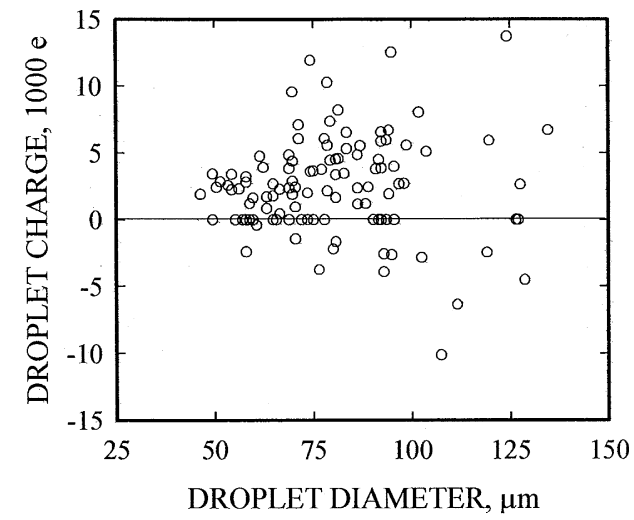

Fig. 3. Distribution of charges as a function of droplet size for distilled water.

(Fig. 4). The similarity in the root-mean-square charges for the droplets from the $\mathrm{NaLSO}_{4}$ and AOT solutions at high concentrations should be noted, however.

Cationic surfactants: The results for dodecylamine hydrochloride (DAH) are presented in Fig. 5 in terms of the RMS and total charges. The general trend for this cationic surfactant 


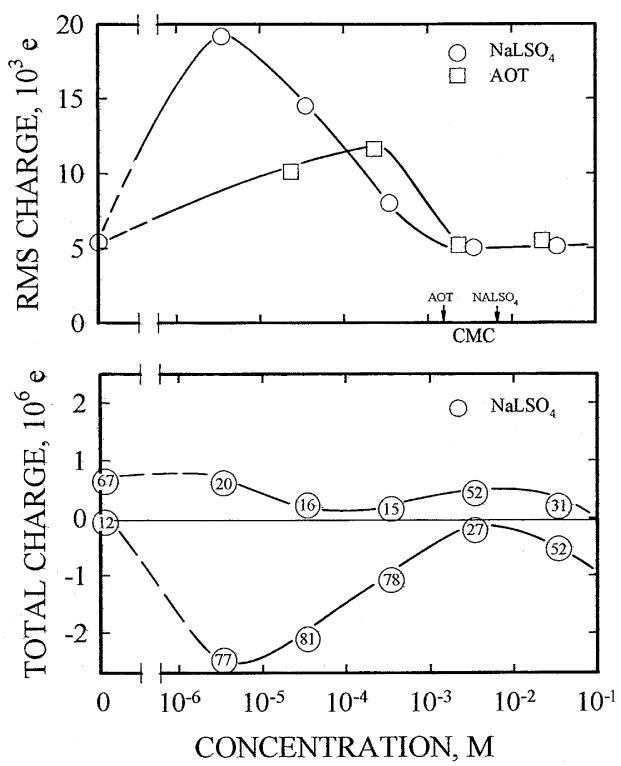

Fig. 4. Charging behavior of the droplets from surfactant solutions: (a) RMS charge of the droplets as a function of Na lauryl sulfate and Aerosol-OT concentration. (b) Total charges on spray droplets as a function of $\mathrm{Na}$ lauryl sulfate concentration. The numbers inside the symbols refer to the percentages of positively and negatively charged droplets in the population.

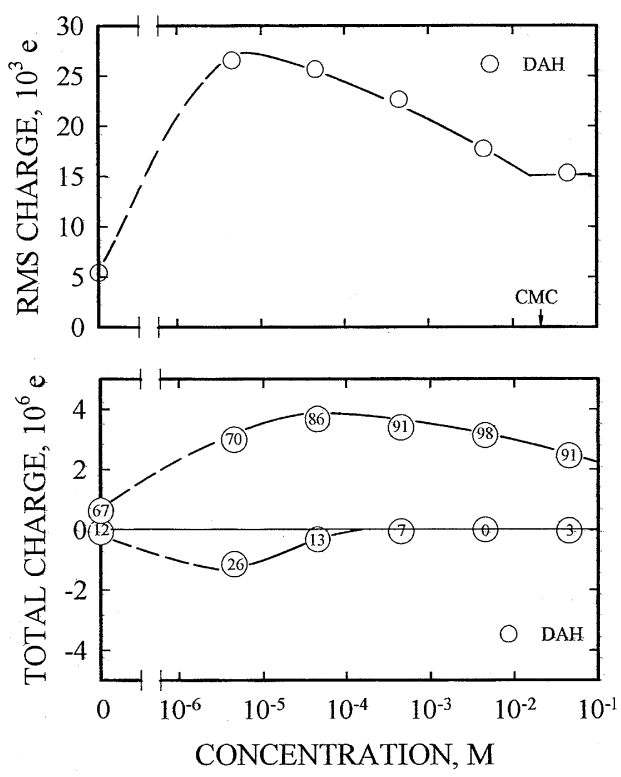

Fig. 5. The RMS and total charges on spray droplets as a function of dodecylamine hydrochloride concentration. The numbers inside the symbols refer to the fractions of positively and negatively charged droplets in the population.

was similar to that observed with the anionic surfactants. The RMS charge displayed an initial increase, but began to decrease with further surfactant addition and seemed to level off at a concentration close to the critical micelle concentration. However, it should be noted that the RMS charges are considerably higher for this cationic surfactant compared to the anionics. It should be noted that the RMS charges are still quite high around the CMC. The negatively charged droplets are almost completely eradicated at high concentrations of this surfactant as seen from their very low percentages. 
The RMS charges obtained with the ethoxylated coco amines are given in Fig. 6. The general behavior is similar to that observed with DAH. The RMS charge goes to a maximum initially before dropping and leveling off at high concentrations. There is one important difference, however. The concentration where maximum RMS charges are observed are very close to the CMC value of these three surfactants.

The RMS charges for C10 in Fig. 6 are given for two repeat set of tests to demonstrate the reproducibility in the data.

Nonionic surfactants: The charging behavior of the nonionic Triton X-100 (TX100) and Triton N-101 (TN101) surfactants was markedly different from that obtained with the ionic surfactants. The RMS charge showed a linear increase with increasing surfactant concentration. Also, the general magnitude of the RMS charges were smaller for these surfactants (Fig. 7). It is important to note that the increase in the RMS charges results from generation of positively charged droplets as seen from the percentages given in the figure.

\section{Physically charged droplets}

A set of experiments was conducted to investigate the response of the charge measurement setup when the charging of the droplets was achieved by applying a potential

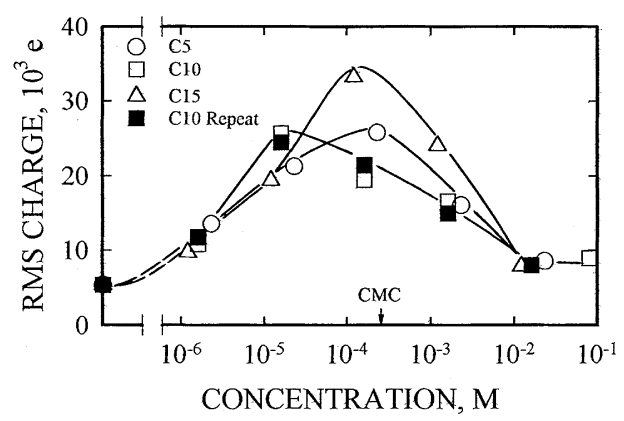

Fig. 6. The RMS and total charges on spray droplets as a function of concentration of C-Series surfactants (the dark symbols refer to the repeat experiments with $\mathrm{C} 10$ ).
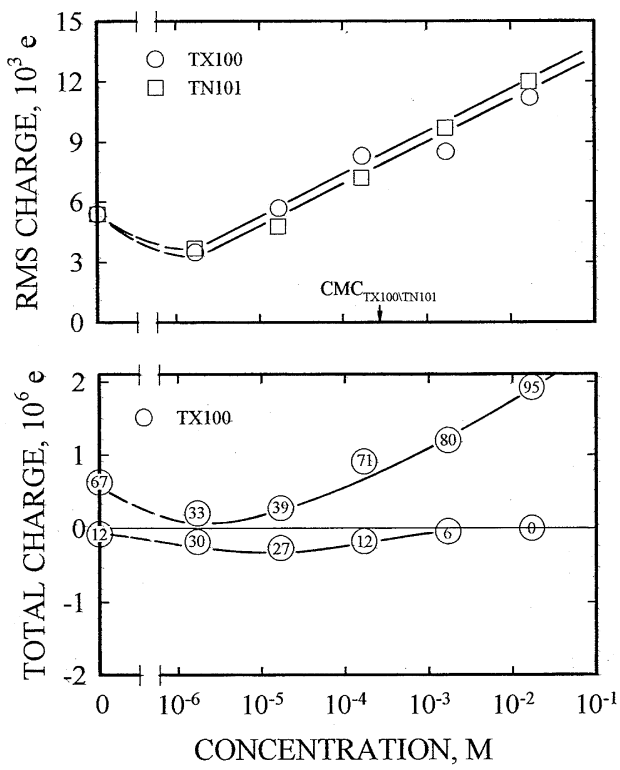

Fig. 7. Charging behavior of the droplets from surfactant solutions (a) RMS charge of the droplets as a function of Triton X-100 and Triton N-101 concentration. (b) Total charges on spray droplets as a function of Triton X-100 concentration. The numbers inside the symbols refer to the fractions of positively and negatively charged droplets in the population. 

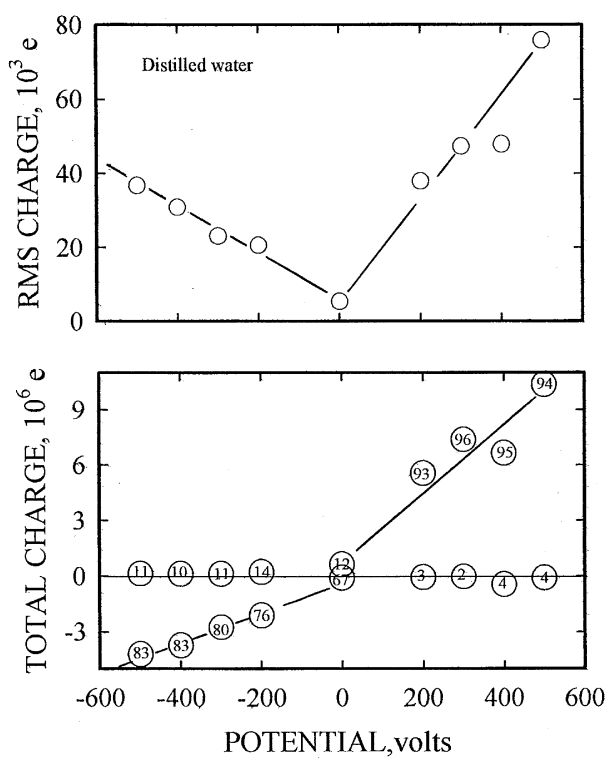

Fig. 8. Total positive and negative charges of the droplets as a function of potential. The numbers on the symbols refer to the fractions of positively and negatively charged droplets in the population.

difference during spraying. Only distilled water was used in the experiments and the droplets were charged by applying positive and negative DC potentials of 200, 300, 400 and 500 volts to the stainless-steel tube attached in front of the spray nozzle. The tube was isolated from the nozzle which was grounded using a plexiglas separator. There was a linear relationship between the applied voltage and the RMS charges observed on the droplets (Fig. 8). The RMS charge of $75.8 \times 10^{3}$ (e) at $+500 \mathrm{~V}$ was the highest observed in all the droplet experiments conducted in this study. The total charges and the percentages of positively and negatively charged droplets were strongly a function of the polarity of the potential applied. Both the percentage of negative droplets and the total negative charge in the system were very low when the applied potential was positive. On the contrary, the fraction of positive droplets and the total positive charge decreased when the applied potential was negative. It should be pointed out that the magnitude of charges observed in the case of positive potential was significantly higher than those obtained at the negative potential. In addition, the percentages of positively charged droplets at negative potentials were always higher than the percentages of the negatively charged droplets at positive potentials.

\section{DISCUSSIONS}

The charges on the distilled water droplets could not be explained alone with the development of a double layer at the air/water interface because of the insufficient number of dissolved ions. Contact electrification is not probable since the spray nozzle was grounded. The magnitude of charging by the free ions in the environment should also be low since the system was saturated with spray droplets for 10 min prior to measurement. It could be that the small contributions from all these effects may add up to result in the charges observed for the distilled water.

The experiments with the ionic surfactants point to a common behavior; Ionic surfactants charge the droplets significantly and the sign of the dominant droplet charge is the same as the sign of the ionic head. It seems that there are three concentration regions of different charge behavior with these surfactants. Initially the RMS charge increases with concentration up to a maximum. It decreases afterwards as more surfactant added and reaches a minimum. The RMS charges become independent of concentration after this point. 
The presence of regions which display different charge characteristics for the ionic surfactants may be explained as follows: The surfactant molecules orient themselves at the interface such that the hydrocarbon chain is expelled out of water while the ionic heads are readily dissolved in it. It is clear that the presence of ionic heads at the interface results in a net contribution to the overall charge of the droplets. As the droplet starts settling in the column, hydrocarbon chains experience a drag force due to the surrounding air molecules and move towards the droplet's upper pole, increasing the packing of surfactant molecules at that location. This effect is known as the Marangoni effect. Close packing of the surfactant molecules on one side of the droplet is accompanied by a diffuse layer of the counter ions extending into the bulk solution in the opposite direction. At this stage such a droplet resembles a dipole. The droplet experiences a force equivalent to the force on an electric dipole in the charge measurement chamber. The accumulation of surfactant molecules on one side of the droplet will become more pronounced, resulting in a stronger dipole interaction with increasing surfactant concentration. Nevertheless, with further surfactant addition, the molecules will gradually occupy all the available sites on the droplet surface. When the total surface coverage is reached, the dipole structure of the droplet will disappear due to uniform distribution of the surfactant molecules around the droplet. At this stage, the counter ions will be distributed uniformly beneath the surfactant layer. This will lead to a decrease in the apparent charge of the droplet due to neutralization of the ionic character of the surfactant molecules. The charge on the droplets will show a decline after a specific concentration value where the dipole effect is most emphasized and will reach a minimum around the total surface coverage concentration where the dipole effect is minimum. Further increase in the surfactant concentration does not bring any appreciable change in the charge of the droplets.

It should be noted that increasing number of hydrophobic tails at the droplet/air interface as the surfactant concentration increases may also have an effect on the droplet charge. The considerable higher charges obtained in the case of cationic surfactants at high concentrations compared to the anionic surfactants suggest the role of hydrocarbon chains for the increase in the positive charges in the system.

For ionic surfactants such as $\mathrm{NaLSO}_{4}$, AOT and DAH where the water solubility is brought about by a simple ion, the charges are always minimum in the vicinity of the CMC (Figs 4 and 5).

For the coco amines the CMC of these surfactants was close to the transition concentration where maximum charges were observed. One explanation is that since coco amines are large surfactant molecules, their diffusion rates and CMC will be significantly lower. At low concentrations surfactant molecules arriving at the interface will directly contribute to the charge. However, charging due to the Marangoni effect will be lower compared to DAH due to slow diffusion of the molecules of this surfactant. The CMC will be reached rather quickly before or around the surface coverage, further slowing the amount of surfactant molecules arriving at the interface. This will result in the observed decrease in the charge. Smaller RMS charges for these surfactants compared to DAH at low concentrations could be due to looser packing of the coco amine molecules having very bulky hydrophilic heads made out of ethylene oxide groups.

The difference in the behavior of the two anionic surfactants, namely $\mathrm{NaLSO}_{4}$ and AOT, can be explained by the differences in their packing at the interface. The sodium $\mathrm{NaLSO}_{4}$ molecule has a hydrophobic tail with a straight-chain structure, whereas the hydrophobic tail of an AOT molecule is branched. Hence, the $\mathrm{NaLSO}_{4}$ molecules will be more closely packed on the droplet's pole compared to the molecules of AOT (Myers, 1988). Since both surfactants have ionic heads similar in character, the charge density per area of droplet will be higher for the densely packed $\mathrm{NaLSO}_{4}$ molecules. This will mean a stronger dipole structure for the droplets of $\mathrm{NaLSO}_{4}$ which will result in higher charge readings. Also, maximum charging will take place at higher concentrations for the surfactant with larger or branched hydrocarbon chains such as AOT due to slow diffusion.

Droplet charges display a steady increase within the concentration range studied for the nonionic surfactants. This behavior seems to be due to an increase in the positive charges in 
the system. The ethylene oxide groups which have a highly polar structure and dissolve in water via hydrogen bonding may also be responsible for the observed increase. Each ethylene oxide group forms a dipole with $\mathrm{CH}_{2}$ as the positive and $\mathrm{O}$ as the negative pole. This results in an induced positive charge on the ethylene oxide groups in their aqueous solutions (Schonfeldt, 1969).

The CMC does not seem to have any effect on the charging behavior of the nonionic surfactants. This is not surprising since both TX100 and TN101 have very small diffusion coefficients, which are in the order of $10^{-7}\left(\mathrm{~cm}^{2} \mathrm{~s}^{-1}\right)$ due to their large ethylene oxide groups. This means that for a $90 \mu \mathrm{m}$ droplet it will take a surfactant molecule in the center about 15-20 s to reach the surface whereas this time is in the order of $2 \mathrm{~s}$ for the ionic surfactants used. Since the charge measurement process is a kinetic event and lasts only about 2-3 s, a complete surface coverage may never be achieved for the droplets of the non-ionic surfactants.

\section{SUMMARY AND CONCLUSIONS}

Based on the findings of this study, the following conclusions could be made:

(1) Surfactants were observed to increase the charge on the spray droplets significantly. The sign and magnitude of charge could be manipulated by varying surfactant type and concentration. This important characteristic of surfactants along with their capacity for changing interfacial properties such as surface tension can be exploited in numerous applications such as spray painting, crop spraying, ink-jet printing, etc.

(2) The sign of the ionic head group determined the droplet charge for ionic surfactants. The charge on the droplets of the cationic surfactant solutions were higher than that on the droplets of the anionic surfactant solutions.

(3) The droplet charge increased initially with increasing surfactant concentration and started to decrease after reaching a maximum for ionic surfactants. It reached a minimum and stayed constant with further surfactant addition. The concentration where minimum charging was observed was around the CMC for the ionic surfactants with simple ionic heads. For ionic surfactants with large head groups, the CMC was in the vicinity of the concentrations where maximum charges were observed. Surfactants with branched hydrocarbon chains showed lesser charges at a given concentration.

(4) For nonionic surfactants the RMS charge showed a steady increase with concentration and did not seem to have any relation to the CMC. The increase in the RMS was mainly due to an increase in the positively charged droplets.

Acknowledgements - The authors acknowledge the support from the Mineral Ínstitutes Program under grant No. G1135142 from the Bureau of Mines, U.S. Department of Interior, as part of the Generic Mineral Technology Center for Respirable Dust, The Pennsylvania State University.

\section{REFERENCES}

Ash, M. and Ash, I. (1992) Industrial Chemical Thesaurus, 2nd Edition, VCH Publishers, Inc., New York. Baily, A. G. (1988) Electrostatic Spraying of Liquids, University of Southampton, England.

Byrne, M. J. (1977) Ph.D. thesis, Natl. Univ. of Ireland.

Chapman, S. (1934) Phys. Rev. 5, 150.

Chapman, S. (1937) Phys. Rev. 52, 184-190.

Chapman, S. (1938a) Phys. Rev. 54, 520-527.

Chapman, S. (1938b) Phys. Rev. 54, 528-533.

Concha, F. and Almendra, E. R. (1979a) Int. J. Mineral Process. 5, 349-367.

Concha, F. and Almendra, E. R. (1979b) Int. J. Mineral Process. 6, 31-41.

Concha, F. and Barrientos, A. (1982) Int. J. Mineral Process. 9, 167-172.

Dodd, E. E. (1952) J. Appl. Phy. 24(1), 73-80.

Hopper, V. D. and Laby, T. H. (1941). Proc. Roy. Soc., A. 178, 243-273.

Hu, Q. (1992) M.S. thesis, The Penn State University.

Gomez, A. (1993) 3rd World Conf. on Experimental Heat Transfer, Fluid Mechanics and Thermodynamics, Elsevier,

Amsterdam, 1, pp. 270.

Iribarne, J. V. and Mason, B. J. (1967) Trans. Faraday Soc. 63, 2234-2245.

Kunkel, W. B. (1950a) J. Appl. Phy. 21, 883-887. 
Kunkel, W. B. (1950b) The Rev. of Sci. Instr. 21, 308-314.

Kunkel, W. B. (1950b) J. Appl. Phy. 21, 820-832.

Lenard, P. (1892) Wied. Ann. 46, 584.

Liu, B. H. Y., Pui, D. Y. H., Kinstley, W. O. and Wayre, G. F. (1987) J. Env. Sci. March-April: 42. Millikan, R. A. (1913) Phy. Rev. 2, 109.

Mohal, B. (1988). PhD. thesis, The Pennsylvania State University.

Mukerjee, P. and Mysels, K. J. (1971) Nat. Stand. Ref. Data Ser., Nat. Bur. Stand. (U.S.) 36, 227.

Myers, D. (1988) Surfactant Science and Technology, VCH Publishers, New York.

Osipow, L. W. (1962) Surface Chemistry, Theory and Industrial Applications. Reinhold Pub. Corp., New York.

Pearse, M. J. and Pope, M. I. (1977). Powder Tech. 17, 83-89.

Polat, H., Hu, Q., Polat, M. and Chander, S. (1993) Proc. 6th US Mine Ventilation Symp., pp. 537-541.

Polat, H., Polat, M., Chander, S. and Hogg, R. (1996) J. Appl. Occup. Envinron. Hyg. 11(2), 817-825.

Reck, R. A. (1967) Nonionic Surfactants. (Edited by M. J. Schick) pp. 187-246. Marcel Dekker, New York.

Schonfeldt, N. (1969) Surface Active Ethylene Oxide Adducts, pp. 133-137. Pergamon Press, New York.

Tang, K. and Gomez, A. (1994a) Phys. Fluids A. 6, 2317.

Tang, K. and Gomez, A. (1994b). J. Aerosol Sci. 25(6), 1237.

Vick, F. A. (1953) British J. Appl. Phys. (Supp.) 2, S1-S5.

White, H. J. (1951) Am. Ins. Electr. Eng. Trans. 70, 1186.

Yamashita, C. and Fen, J. B. (1984) J. Phys. Chem. 88, 4671. 